\title{
QOE Based Heterogeneous Network Selection Using Pythagorean Fuzzy Methods
}

\author{
Lei Zhu, Kaixin Cheng $\mathbb{D}^{D}$, Lei Wang $\mathbb{D}$, and Wei Tong \\ College of Communications Engineering, Army Engineering University of PLA, Nanjing 210000, China \\ Correspondence should be addressed to Kaixin Cheng; ckx_paper@outlook.com
}

Received 25 July 2018; Revised 10 September 2018; Accepted 12 September 2018; Published 28 October 2018

Academic Editor: Anna M. Gil-Lafuente

Copyright (C) 2018 Lei Zhu et al. This is an open access article distributed under the Creative Commons Attribution License, which permits unrestricted use, distribution, and reproduction in any medium, provided the original work is properly cited.

\begin{abstract}
Considering the diversity of demands in heterogeneous network selection problem, we combine Choquet integral with traditional aggregation operators in Pythagorean fuzzy environment, inventing kinds of new operators based on Choquet integral, including PFCOWA (as well as RP-PFOWA) operator, PFCWA operator, PFCOWG (as well as RP-PFOWG) operator, and PFCWG operator, which are capable of solving different attributes relationship prioritized situations. Then, transferring relationship and properties of the operators are further discussed. Additionally, to overcome the weakness of traditional method of evaluation, a novel evaluating system of QOE in fuzzy environment is proposed. Based on these works, an example of heterogeneous network selection is given to illustrate the validity of this method.
\end{abstract}

\section{Introduction}

Pythagorean fuzzy set (PFS) is an extension of intuitionistic fuzzy set (IFS) [1-3] proposed by Yager [4-6]. Intuitionistic fuzzy set [1] describes the imprecise information using two indicators: membership and nonmembership degree. When an expert judges if an alternative is up to standard, the answer can be hard to say by merely yes or no. By using IFS, he can give a membership degree which between 0 and 1 to describe the degree conforms to the standard and vice versa. An important condition is that the total of two membership indicators should not be exceeding one. However, this condition limits the applied range of IFS. Therefore, Yager puts forward PFS to work out the situation when the accumulating of two indicators value is exceeding one while the square sum of them is less than one. Obviously, IFS is the special case of PFS, where the latter one can be more widely used when describing the imprecise and vague information. For the range of membership degree is extended, PFS can express subtler uncertainties than IFS. Since PFS has been developed, the study on it never stopped [7-12].

Zhang and $\mathrm{Xu}$ [7] defined some operation and the score function of PFSs. Yager $[4,6]$ proposed some basic aggregation operators including PFWA operator and PFWG operator. Later, various types of operators [8] are developed in Pythagorean environment, including the PFOWA operator, the GPFOWA operator, the PFOWG operator, and the GPFOWG operator. Most of these operators have been used on solving MADM problems. Though many Pythagorean fuzzy aggregation operators had been developed, most of them only consider the elements in fuzzy sets as independent, ignoring the situation when interaction exists between attributes.

However, except single attribute performance, correlation between those attributes is more important in some circumstances. Ji and Wang [13] established a new method for selecting TPL providers under single-valued neutrosophic environments, which simultaneously takes into account the interrelationships among criteria. The single-valued neutrosophic hesitant fuzzy geometric weighted Choquet integral Heronian mean operator [14], which is based on the Heronian mean and Choquet integral, is proposed.

The application of Choquet integral in fuzzy operators [15-20] is not an emerging idea. The Choquet integral [21] can be used to illustrate the interaction of considered attributes, which is an available tool to measure the intended effect of indefinite event. Yager $[15,16]$ introduced an inducing factor into Choquet aggregating process, getting a bright new 
operator: I-COA operator. In intuitionistic fuzzy environment, $\mathrm{Xu}$ [17] expended some information aggregating methods by means of the Choquet integral. Tan and Chen $[19,20]$ strengthened the preference relations of decision-makers by using the I-COA operator in group decision-making. Moreover, Xu and Xia [18] applied Choquet integral to broaden applied scene of former operators. From previous study we can see the feasibility and necessity of transporting this method to Pythagorean environment. So we introduce fuzzy measure into Pythagorean fuzzy operators, acquiring the relationship prioritized Pythagorean aggregation fuzzy operators [22], while fuzzy measure is the basic of Choquet integral.

How to evaluate the subjective standard is a tough work. Usually, attributes put into consideration include throughput, delay, jitter, and cost. Those are decisive factors of network QOE (quality of experience), which has arouse the attention of the academic community as well as becoming one of the major evaluation indicators on industry. The concept of QOE is born out of QOS (quality of service), but it focuses on the subjective feelings of users, which meets the need of humanization. Reference [23] introduces the existing QOE models and evaluation methods, and different disciplines have their own emphasis on the structure of the evaluation system. Therefore, this paper proposes a brand-new QOE evaluation system based on Pythagorean fuzzy numbers combined with the heterogeneous network environment and the characteristics of services.

This paper further proposes some Pythagorean fuzzy operators, including PFCOWA operator, PFCWA operator, and PFCWG operator, based on our previous work, and unifies their designations and properties, which are capable of solving different attributes relationship prioritized situations. Then, transferring relationship and properties of the operators are further discussed. Additionally, a novel objective evaluating system of QOE in fuzzy environment is proposed. Ultimately, based on the proposed evaluation system, different operators are applied in aggregating information for MADM problems on network selection.

\section{Basic Knowledge Review}

Before formally introducing newly developed operators, it is necessary to recall some fundamental definition and properties.

\subsection{Pythagorean Fuzzy Numbers}

Definition 1 (see [4-7]). $\beta=P\left(\mu_{\beta}, v_{\beta}\right)$ is Pythagorean fuzzy number in which $\mu_{p}, v_{p}$ separately represents the membership degree and the nonmembership degree. $\mu_{\beta}, v_{\beta} \in[0,1]$, $\pi_{\beta}=\sqrt{1-\left(\mu_{\beta}\right)^{2}-\left(\nu_{\beta}\right)^{2}}$ is the degree of uncertainty, and $\left(\mu_{\beta}\right)^{2}+\left(\nu_{\beta}\right)^{2} \leq 1$.

Zhang and $\mathrm{Xu}$ [7] defined the following operations.

Definition 2 (see [7]). For three PFNs $\beta=P\left(\mu_{\beta}, v_{\beta}\right), \beta_{1}=$ $P\left(\mu_{\beta_{1}}, v_{\beta_{1}}\right), \beta_{2}=P\left(\mu_{\beta_{2}}, v_{\beta_{2}}\right)$, they have the operations rules as follows:
(1) $\beta_{1} \oplus \beta_{2}=P\left(\sqrt{\mu_{\beta_{1}}^{2}+\mu_{\beta_{2}}^{2}-\mu_{\beta_{1}}^{2} \mu_{\beta_{2}}^{2}}, v_{\beta_{1}} v_{\beta_{2}}\right)$.

(2) $\beta_{1} \otimes \beta_{2}=P\left(\mu_{\beta_{1}} \mu_{\beta_{2}}, \sqrt{v_{\beta_{1}}^{2}+v_{\beta_{2}}^{2}-v_{\beta_{1}}^{2} v_{\beta_{2}}^{2}}\right)$

(3) $\lambda \beta=P\left(\sqrt{1-\left(1-\mu_{\beta}^{2}\right)^{\lambda}},\left(\nu_{\beta}\right)^{\lambda}\right), \lambda>0$.

(4) $\beta^{\lambda}=P\left(\left(\mu_{\beta}\right)^{\lambda}, \sqrt{1-\left(1-v_{\beta}^{2}\right)^{\lambda}}\right), \lambda>0$.

Obviously, the results of operations are still PFNs. They have the following properties:

(1) $\beta_{1} \oplus \beta_{2}=\beta_{2} \oplus \beta_{1}$.

(2) $\beta_{1} \otimes \beta_{2}=\beta_{2} \otimes \beta_{1}$.

(3) $\lambda\left(\beta_{1} \oplus \beta_{2}\right)=\lambda \beta_{1} \oplus \lambda \beta_{2}, \lambda>0$.

(4) $\left(\beta_{1} \otimes \beta_{2}\right)^{\lambda}=\beta_{1}{ }^{\lambda} \otimes \beta_{2}{ }^{\lambda}, \lambda>0$.

(5) $\left(\lambda_{1}+\lambda_{2}\right) \beta=\lambda_{1} \beta \oplus \lambda_{2} \beta, \lambda_{1}, \lambda_{2}>0$.

(6) $\beta^{\lambda_{1}+\lambda_{2}}=\beta^{\lambda_{1}} \otimes \beta^{\lambda_{2}}, \lambda_{1}, \lambda_{2}>0$.

2.2. Fuzzy Measure. To apply Choquet integral into Pythagorean fuzzy operators, the concept of fuzzy measure [24-26] should be firstly elaborated.

Definition 3 (see [24-26]). Fuzzy measure $F_{m}$ on finite space $S$ is a mapping $F_{m}: \vartheta[S] \longrightarrow[0,1]$, which meets the following conditions:

(1) $F_{m}[\varnothing]=0, F_{m}[S]=1$.

(2) For all $A, B \subseteq S$ and $A \subseteq B, F_{m}(A) \leq F_{m}(B)$.

(3) $F_{m}(A \cup B)=F_{m}(A)+F_{m}(B)+\rho F_{m}(A) F_{m}(B)$, for all $A, B \subseteq S$, and $A \cap B=\varnothing, \rho \in(-1, \infty)$.

Parameter $\rho$ has its meaning as follows:

$\rho=0$ signifies that the attributes under consideration are dependent. $\rho>0$ shows there is mutual promotion between the attributes, while $\rho<0$ means the attributes weaken each other when being aggregated.

For this reason, this mathematical tool can be used to illustrate relationship between several attributes for multiattribute decision-making problems. The details for how to determine value of the parameters have been discussed in [22]. The application of fuzzy measure as well as Choquet integral in aggregation operators has been proved valid, which brings out a relationship preference strategy distinguished with PROMETHEE. To acquire properties of the operator and further develop some other equipment for different multiattributes problems, we redefine a kind of aggregation operator: Pythagorean fuzzy operators based on Choquet integral. The relationship prioritized operators belong to this category. Besides, it also contains some promotion form operators with more integration traits. Next, we will introduce them in detail.

\section{Pythagorean Fuzzy Choquet Operators}

In this chapter, we utilize the Choquet integral into Pythagorean fuzzy environment, developing a series of neoteric operators. The properties of these operators and the 
relationship between them are further discussed. Meanwhile, according to different aggregation attributes of these operators, we analyze the using scenes of them, which are their applied environment.

\subsection{Pythagorean Fuzzy Choquet Averaging (PFCA) Operators}

Definition 4. Pythagorean fuzzy Choquet order weighted averaging (PFCOWA) operator. [22]

$\beta_{i}$ are PFNs, $S:\left\{\beta_{i}=P\left(\mu_{\beta_{i}}, v_{\beta_{i}}\right)\right\}, i=1,2, \ldots, n . s_{i}$ is the ith largest in $\beta_{i}$, and set $L_{i}$ as a subset of $S$ :

$$
\begin{aligned}
& L_{0}=\varnothing \\
& L_{i}=\left\{\sum_{k=1}^{i} s_{k}\right\} .
\end{aligned}
$$

The Pythagorean fuzzy Choquet ordered weighted averaging operator is defined as

$$
\begin{aligned}
& \operatorname{PFCOWA}\left(\beta_{1}, \beta_{2}, \ldots, \beta_{n}\right) \\
& =\bigoplus_{i=1}^{n}\left(F_{m}\left(L_{i}\right)-F_{m}\left(L_{i-1}\right)\right) s_{i},
\end{aligned}
$$

$F_{m}$ is the fuzzy measure on $S$. According to the operational rules, and the PFCOWA operator can be expressed as below:

$$
\begin{aligned}
& \operatorname{PFCOWA}\left(\beta_{1}, \beta_{2}, \ldots, \beta_{n}\right)=\bigoplus_{i=1}^{n}\left(F_{m}\left(L_{i}\right)\right. \\
& \left.-F_{m}\left(L_{i-1}\right)\right) s_{i} \\
& =P\left(\sqrt{1-\prod_{i=1}^{n}\left(1-\mu_{s_{i}}^{2}\right)^{\left(F_{m}\left(L_{i}\right)-F_{m}\left(L_{i-1}\right)\right)}},\right. \\
& \left.\prod_{i=1}^{n} v_{s_{i}}\left(F_{m}\left(L_{i}\right)-F_{m}\left(L_{i-1}\right)\right)\right) \text {. }
\end{aligned}
$$

The result is still PFN. And the operator has the following properties.

(1) Commutativity. $\operatorname{PFCOWA}\left(\beta_{1}, \beta_{2}, \ldots, \beta_{n}\right)=$ $\operatorname{PFCOWA}\left(\beta_{(1)}, \beta_{(2)}, \ldots, \beta_{(n)}\right),\left(\beta_{(1)}, \beta_{(2)}, \ldots, \beta_{(n)}\right)$ is an arbitrary sort of $\left(\beta_{1}, \beta_{2}, \ldots, \beta_{n}\right)$.

Proof. This conclusion is easy to prove, for the operator will reorder the set by its value, and the result is the same for one fixed set no matter what the original order is.

(2) Boundedness. Set $\beta_{\min }$ as the smallest value in $\left(\beta_{1}, \beta_{2}, \ldots, \beta_{n}\right)$, while $\beta_{\max }$ is the largest. We will have the property that

$$
\beta_{\min } \leq \operatorname{PFCOWA}\left(\beta_{1}, \beta_{2}, \ldots, \beta_{n}\right) \leq \beta_{\max } .
$$

Proof.

$$
\begin{aligned}
& \operatorname{PFCOWA}\left(\beta_{1}, \beta_{2}, \ldots, \beta_{n}\right) \\
& =\bigoplus_{i=1}^{n}\left(F_{m}\left(L_{i}\right)-F_{m}\left(L_{i-1}\right)\right) s_{i} \\
& \leq \bigoplus_{i=1}^{n}\left(F_{m}\left(L_{i}\right)-F_{m}\left(L_{i-1}\right)\right) \beta_{\max }, \\
& \text { For } \bigoplus_{i=1}^{n}\left(F_{m}\left(L_{i}\right)-F_{m}\left(L_{i-1}\right)\right)=1, \\
& \operatorname{PFCOWA}\left(\beta_{1}, \beta_{2}, \ldots, \beta_{n}\right) \leq \beta_{\max } .
\end{aligned}
$$

Similarly, $\beta_{\min } \leq \operatorname{PFCOWA}\left(\beta_{1}, \beta_{2}, \ldots, \beta_{n}\right)$, and the conclusion is successfully proved.

(3) Monotonicity. The PFCOWA operator is monotonically increasing based on value of argument value. If $\beta_{i} \leq \beta_{i}^{\prime}$ is true for each $i=1,2, \ldots, n$, then

$$
\begin{aligned}
& \operatorname{PFCOWA}\left(\beta_{1}, \beta_{2}, \ldots, \beta_{n}\right) \\
& \quad \leq \operatorname{PFCOWA}\left(\beta_{1}^{\prime}, \beta_{2}^{\prime}, \ldots, \beta_{n}^{\prime}\right) .
\end{aligned}
$$

Proof. Take the derivative of the operator with respect to argument value $\beta$.

$$
\begin{aligned}
& \frac{\partial \operatorname{PFCOWA}\left(\beta_{1}, \beta_{2}, \ldots, \beta_{n}\right)}{\partial \beta_{i}}=F_{m}\left(L_{i}\right)-F_{m}\left(L_{i-1}\right) \\
& \quad \geq 0 .
\end{aligned}
$$

Apparently, the result $F_{m}\left(L_{i}\right)-F_{m}\left(L_{i-1}\right)$ is constantly greater than or equal to zero according to the definition. Therefore, the conclusion is proved.

(4) Idempotency. If $\beta_{i}=\beta_{0}$ is true for each $i=1,2, \ldots, n$, then

$$
\operatorname{PFCOWA}\left(\beta_{1}, \beta_{2}, \ldots, \beta_{n}\right)=\beta_{0}
$$

This property can be easily proved by the definition and operation rules.

Definition 5. Pythagorean fuzzy Choquet weighted averaging (PFCWA) operator.

$S$ is a collection of PFNs, $S:\left\{\beta_{i}=P\left(\mu_{\beta_{i}}, \nu_{\beta_{i}}\right)\right\}$, where $i=1,2, \ldots, n . F_{m}$ is the fuzzy measure on $S$, and set $L_{i}^{\prime}$ as a subset of $S$. It meets the following regulations:

$$
\begin{aligned}
& L_{0}^{\prime}=\varnothing \\
& L_{i}^{\prime}=\left\{\sum_{k=1}^{i} \beta_{k}\right\} .
\end{aligned}
$$


The PFCWA operator is

$$
\begin{aligned}
& \operatorname{PFCWA}\left(\beta_{1}, \beta_{2}, \ldots, \beta_{n}\right) \\
& =P\left(\sum_{i=1}^{n}\left(F_{m}\left(L_{i}^{\prime}\right)-F_{m}\left(L_{i-1}^{\prime}\right)\right) \mu_{\beta_{i}},\right. \\
& \left.\sum_{i=1}^{n}\left(F_{m}\left(L_{i}^{\prime}\right)-F_{m}\left(L_{i-1}^{\prime}\right)\right) v_{\beta_{i}}\right) .
\end{aligned}
$$

Furthermore, we take some special occasions into consideration.

(1) When $F_{m}(S)=\sum_{s_{i} \in S} F_{m}\left(\left\{s_{i}\right\}\right)$, which means elements in the set are independent, then, the weighted vector is $\omega_{i}=F_{m}\left(L_{i}\right)-F_{m}\left(L_{i-1}\right)=F_{m}\left(s_{i}\right)$ and $\sum_{i=1}^{n} \omega_{i}=1, s_{i}$ is the ith largest in $\beta_{i}$, and formulas (2) and (3) reduce to

$$
\begin{aligned}
& \operatorname{PFCOWA}\left(\beta_{1}, \beta_{2}, \ldots, \beta_{n}\right)=\bigoplus_{i=1}^{n} F_{m}\left(s_{i}\right) s_{i}=\bigoplus_{i=1}^{n} \omega_{i} s_{i} \\
& \quad=P\left(\sqrt{\left.1-\prod_{i=1}^{n}\left(1-\mu_{s_{i}}^{2}\right)^{\omega_{i}}, \prod_{i=1}^{n} v_{s_{i}}{ }^{\omega_{i}}\right)}\right. \\
& =\operatorname{PFOWA}\left(\beta_{1}, \beta_{2}, \ldots, \beta_{n}\right) .
\end{aligned}
$$

The PFCOWA operator is transformed to PFOWA [8]. Especially, when $\omega_{i}=F_{m}\left(s_{i}\right)=1 / n$, the operator continues to be simplified to PFA operator.

(2) When $F_{m}(S)=\sum_{s_{i} \in S} F_{m}\left(\left\{s_{i}\right\}\right), \omega_{i}=F_{m}\left(L_{i}^{\prime}\right)-$ $F_{m}\left(L_{i-1}^{\prime}\right)=F_{m}\left(\beta_{i}\right)$, then, formula (10) reduces to

$$
\begin{aligned}
& \operatorname{PFCWA}\left(\beta_{1}, \beta_{2}, \ldots, \beta_{n}\right)=P\left(\sum_{i=1}^{n} \omega_{i} \mu_{\beta_{i}}, \sum_{i=1}^{n} \omega_{i} \nu_{\beta_{i}}\right) \\
& \quad=\operatorname{PFWA}\left(\beta_{1}, \beta_{2}, \ldots, \beta_{n}\right) .
\end{aligned}
$$

The PFCWA operator becomes PFWA [8]. Especially, when $\omega_{i}=1 / n$, the operator continues to be simplified to PFA operator.

Properties of PFCWA operator are quite similar to that of PFCWOA operator except commutativity. Proving process is in accordance with the former operator.

(1) Boundedness. Set $\beta_{\min }$ as the smallest value in $\left(\beta_{1}, \beta_{2}\right.$, $\ldots, \beta_{n}$ ), while $\beta_{\max }$ is the largest. We will have the property that

$$
\beta_{\min } \leq \operatorname{PFCWA}\left(\beta_{1}, \beta_{2}, \ldots, \beta_{n}\right) \leq \beta_{\max } .
$$

(2) Monotonicity. The PFCWA operator is monotonically increasing based on value of argument value. If $\beta_{i} \leq \beta_{i}^{\prime}$, $(i=1,2, \ldots, n)$, then

$$
\begin{aligned}
& \operatorname{PFCWA}\left(\beta_{1}, \beta_{2}, \ldots, \beta_{n}\right) \\
& \quad \leq \operatorname{PFCOWA}\left(\beta_{1}^{\prime}, \beta_{2}^{\prime}, \ldots, \beta_{n}^{\prime}\right) .
\end{aligned}
$$

(3) Idempotency. When $\beta_{i}=\beta_{0}$, for every $i=1,2, \ldots, n$, then

$$
\operatorname{PFCWA}\left(\beta_{1}, \beta_{2}, \ldots, \beta_{n}\right)=\beta_{0} \text {. }
$$

\subsection{Pythagorean Fuzzy Choquet Geometric (PFCG) Operators}

Definition 6. Pythagorean fuzzy Choquet order weighted geometric (PFCOWG) operator.

$S$ is a collection of PFNs, $S:\left\{\beta_{i}=P\left(\mu_{\beta_{i}}, v_{\beta_{i}}\right)\right\}$, where $i=1,2, \ldots, n$. $s_{i}$ has the ith largest value in $\beta_{i}$, and set $L_{i}$ as a subset of $S$; it meets the following requirements:

$$
\begin{aligned}
& L_{0}=\varnothing \\
& L_{i}=\left\{\sum_{k=1}^{i} s_{k}\right\} .
\end{aligned}
$$

The Pythagorean fuzzy Choquet order weighted geometric operator is defined as follows:

$$
\operatorname{PFCOWG}\left(\beta_{1}, \beta_{2}, \ldots, \beta_{n}\right)=\bigotimes_{i=1}^{n} s_{i}{ }^{\left(F_{m}\left(L_{i}\right)-F_{m}\left(L_{i-1}\right)\right)} \text {, }
$$

$F_{m}$ is the fuzzy measure on $S$; according to the operations of PFNs in Definition 2(3), the PFCOWA operator has form as below:

$$
\begin{aligned}
& \operatorname{PFCOWG}\left(\beta_{1}, \beta_{2}, \ldots, \beta_{n}\right)=\bigotimes_{i=1}^{n} x_{i}^{\left(F_{m}\left(L_{i}\right)-F_{m}\left(L_{i-1}\right)\right)} \\
& =P\left(\prod_{i=1}^{n} \mu_{s_{i}}{ }^{\left(F_{m}\left(L_{i}\right)-F_{m}\left(L_{i-1}\right)\right)},\right. \\
& \sqrt{\left.1-\prod_{i=1}^{n}\left(1-v_{s_{i}}^{2}\right)^{\left(F_{m}\left(L_{i}\right)-F_{m}\left(L_{i-1}\right)\right)}\right)} .
\end{aligned}
$$

The result of aggregation is still PFNs.

Definition 7. Pythagorean fuzzy Choquet weighted geometric (PFCWG) operator.

$S$ is a collection of PFNs, $S:\left\{\beta_{i}=P\left(\mu_{\beta_{i}}, \nu_{\beta_{i}}\right)\right\}$, where $i=1,2, \ldots, n . F_{m}$ is the fuzzy measure on $S$, and set $L_{i}^{\prime}$ as a subset of $S$; it meets the following regulations:

$$
\begin{aligned}
L_{0}^{\prime} & =\varnothing \\
L_{i}^{\prime} & =\left\{\sum_{k=1}^{i} \beta_{k}\right\}
\end{aligned}
$$

The PFCWG operator is

$$
\begin{aligned}
& \operatorname{PFCWG}\left(\beta_{1}, \beta_{2}, \ldots, \beta_{n}\right) \\
& \quad=P\left(\prod_{i=1}^{n} \mu_{\beta_{i}}\left(F_{m}\left(L_{i}^{\prime}\right)-F_{m}\left(L_{i-1}^{\prime}\right)\right), \prod_{i=1}^{n} \nu_{\beta_{i}}\left(F_{m}\left(L_{i}^{\prime}\right)-F_{m}\left(L_{i-1}^{\prime}\right)\right)\right) .
\end{aligned}
$$

Also, we take some special occasions into consideration:

(1) When it meets formula (17), which is $F_{m}(S)=$ $\sum_{s_{i} \in S} F_{m}\left(\left\{s_{i}\right\}\right)$, then, the weighted vector $\omega_{i}=F_{m}\left(L_{i}\right)-$ $F_{m}\left(L_{i-1}\right)=F_{m}\left(s_{i}\right)$ and $\sum_{i=1}^{n} \omega_{i}=1, s_{i}$ has the ith 
largest value in $\beta_{i}$, and formulas (17) and (18) reduce to

$$
\begin{aligned}
& \operatorname{PFCOWG}\left(\beta_{1}, \beta_{2}, \ldots, \beta_{n}\right)=\bigotimes_{i=1}^{n} s_{i}{ }^{F_{m}\left(s_{i}\right)}=\bigotimes_{i=1}^{n} s_{i}{ }^{\omega_{i}} \\
& \quad=P\left(\prod_{i=1}^{n} \mu_{s_{i}}{ }^{\omega_{i}}, \sqrt{1-\prod_{i=1}^{n}\left(1-v_{s_{i}}^{2}\right)^{\omega_{i}}}\right) \\
& =\operatorname{PFOWG}\left(\beta_{1}, \beta_{2}, \ldots, \beta_{n}\right) .
\end{aligned}
$$

The PFCOWG operator is transformed to PFOWG [8]. Especially, when $\omega_{i}=F_{m}\left(s_{i}\right)=1 / n$, the operator continues to be simplified to PFOWG operator.

(2) When it meets formula (17), $F_{m}(S)=\sum_{s_{i} \in S} F_{m}\left(\left\{s_{i}\right\}\right)$, $\omega_{i}=F_{m}\left(L_{i}^{\prime}\right)-F_{m}\left(L_{i-1}^{\prime}\right)=F_{m}\left(s_{i}\right)$, and, then, formula (20) reduces to

$$
\operatorname{PFCWG}\left(\beta_{1}, \beta_{2}, \ldots, \beta_{n}\right)=P\left(\bigotimes_{i=1}^{n} \mu_{\beta_{i}}{ }^{\omega_{i}}, \bigotimes_{i=1}^{n} v_{\beta_{i}}{ }^{\omega_{i}}\right) \text {. }
$$

The PFCWG operator becomes PFWG [8].

\section{Heterogeneous Network Selection}

Operators proposed above are distinct from those of PROMETHEE, taking relationship between attributes into consideration as weighting coefficient, which affects the selection of alternatives. These operators provide fast and effective method with heterogeneous wireless network accessing selection problem, for it is a challenge to decide which network to access when alternatives are multiple, while business needs are various. Therefore, accessing decisions focusing on a particular attribute becomes no longer applicable. According to different business need, the selection standards varying with each other for a business can be sensitive to several attributes at the same time. While satisfying the attribute value requirements, it is also necessary to consider whether attributes needed can be achieved at the same time, which is the relationship between attributes. In brief, the core strategy is to find out the alternative with the best compromise of attribute value and relationship.

4.1. QOE Evaluation System. Considering that it is an evaluation index based on user experience, QOE has inherent fuzzy characteristics. Therefore, this paper uses the Pythagorean fuzzy number to evaluate the performance of different networks and their attributes. In the past, experts scoring method is the main source of data. Though it has accuracy, it is also time-consuming and labor-intensive, with low portability and real-time capability, which is unsuitable as a necessary preparation for decision-making problem. So, it is significant to develop an objective attribute data preprocessing system which is highly portable and real-time.

(1) Determination of QOE. There are many ways to evaluate QOE. In this paper, we use the improved type scaling method.
Traditional method merely divides QOE into two categories, acceptable and unacceptable. Though it can be easily done, the process is over-rough, which can not express user willing and network performance exactly and scientifically. To overcome this shortcoming, this part we introduce Pythagorean fuzzy number into evaluation. It can use membership and nonmembership of PFNs to describe the degree which the attributes categories conform to. For example, the QOE of a specific business in heterogeneous wireless network is either acceptable or unacceptable in traditional judging method. The improved one gives a PFN $(0.82,0.19)$ as a result, which means the degree of acceptability of the network for this business is 0.82 , while 0.19 is the degree of unacceptability. As a result, the improved method expresses the inherent fuzzy characteristic of QOE, describing users' subjective feelings in detail at the same time.

(2) Fuzzification. The process of transferring attribute value to fuzzy numbers, as well as the determination of membership and nonmembership degree, is named fuzzification. At present, there are mainly two methods, subjective evaluation and objective evaluation. The subjective evaluation method is to obtain the fuzzy decision matrix for judgment of each network attribute through expert scoring and user feedback. The advantage of this method is its high accuracy, which can directly express the user's feelings. However, the disadvantages are also obvious. The scoring process is inefficient, while portability and real-time performance are both poor. Objective evaluation method is based on attribute characteristics, distinguishing cost-type, and benefit-type attributes and obtains the corresponding user experience quality value by conforming to the actual QOE mapping function. This method is objective and fair, with strong portability and good real-time performance, while the strain capacity is weak, and the accuracy of the results is difficult to determine.

In this paper, we adopt these two methods at the same time, contrasting the results to search for the better choice in this experiment circumstance. Figure 1 is the QOE evaluation system.

4.2. Experiment. Based on the experiment environment in [27], we use proposed preprocessing system to deal with the data and then aggregate values by developed operators to provide selecting strategy. Comparison between different operators will be displayed in chart.

(1) Experiment Environment. The heterogeneous wireless network consists of UMTS, 802.11.a, 802.11.b, and WIMAX. The business includes session, stream, interaction, and background. Different business has different degree of sensitivity toward the same attribute. For example, the business of flow is quite sensitive on packet loss rate and bit error, while it is tolerant on real-time indicators. However, the session business is the opposite. In this experiment, we take session business as example to show the whole process explicitly. Some variable descriptions are illustrated in Table 1.

(2) Data Preprocessing. The original data of networks in various attributes in Table 2 is based on [27]. 


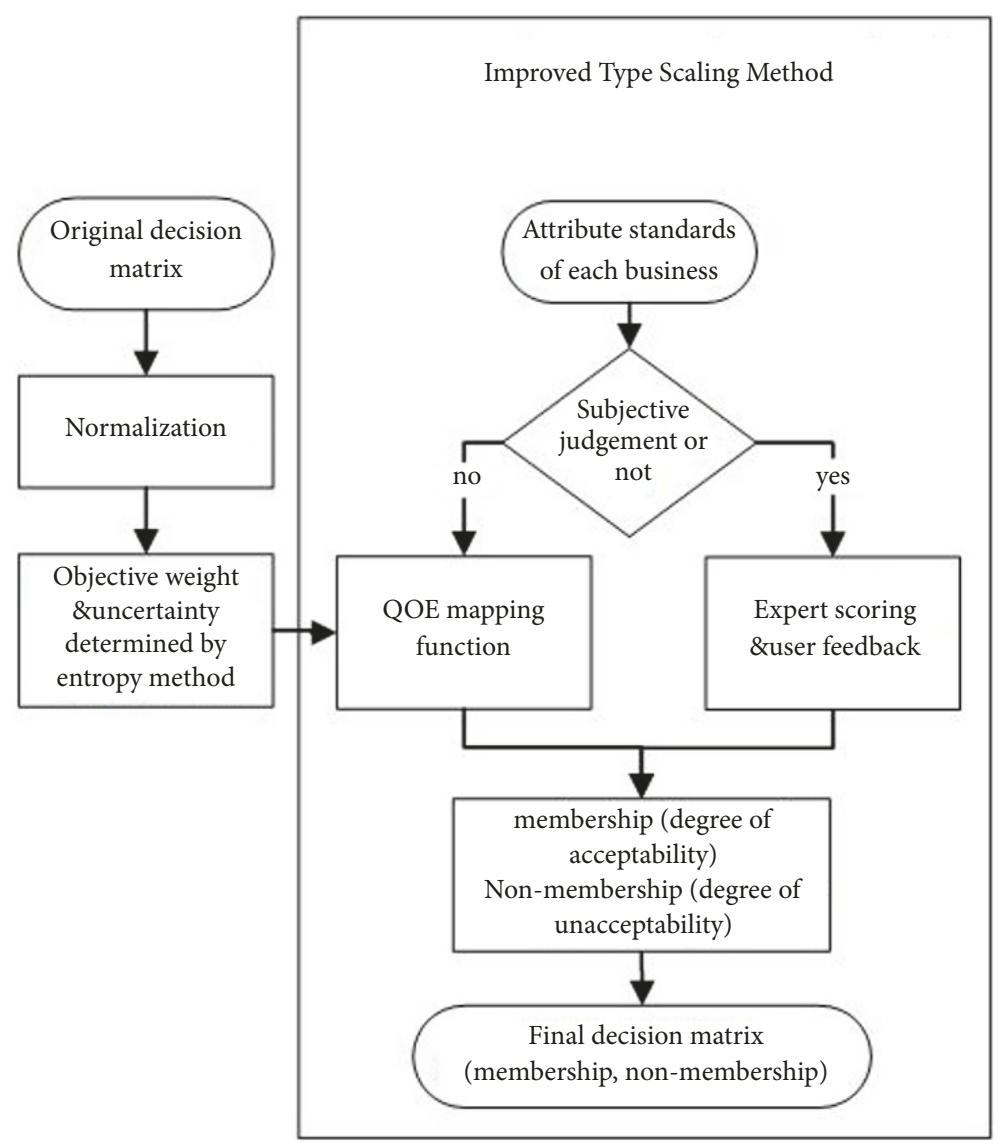

FIGURE 1: QOE evaluation system.

TABLE 1: Variable description.

\begin{tabular}{lc}
\hline Variables & Descriptions \\
\hline$N_{i}(i=1,2,3,4)$ & set of all networks \\
\hline$Q_{j}(j=1,2,3,4,5)$ & set of all attributes \\
\hline$X=\left\{x_{i j}\right\}$ & original decision matrix \\
\hline$E_{j}$ & information entropy of attribute $j$ \\
\hline$R=\left\{r_{i j}\right\}$ & normalized decision matrix \\
\hline$\pi_{j}$ & uncertainty of attribute $j$ \\
\hline$\omega_{o b j}$ & objective weight vector \\
\hline$\left\{\beta_{i j}^{o b j}\right\}$ & final decision matrix by objective method \\
\hline$\left\{\beta_{i j}^{\text {sub }}\right\}$ & final decision matrix by subjective method \\
\hline
\end{tabular}

Because the units of different attributes are not uniform, they need to be normalized firstly. The normalized decision matrix is $R=\left\{r_{i j}\right\}$, and $r_{i j}=x_{i j} / \sqrt{\sum_{i=1}^{m}\left(x_{i j}\right)^{2}}, i=1, \ldots$, $m ; j=1, \ldots, n$.. The result is displayed in Table 3 .

Next, the objective weight and uncertainty will be learned through network attributes value. Entropy evaluation method [28] is a regular one in detecting objective weight of network attributes, the principle of which is the statement in information theory about entropy. When the degree of disorder in a system increases, entropy rises when information of system decreases. Applied to weight acquirement, it endows attributes which have low information with low weight and those high information attributes with higher weights.

(1) Calculate information entropy $p_{i j}$ of network attributes:

$$
\begin{aligned}
E_{j} & =\frac{\sum_{i=1}^{m} p_{i j} \ln p_{i j}}{\ln m}, \quad j=1,2,3, \ldots, n \\
p_{i j} & =\frac{r_{i j}}{\sum_{k=1}^{m} r_{k j}}, \quad i=1,2,3, \ldots, m ; j=1,2,3, \ldots, n
\end{aligned}
$$

(2) Determine the uncertainty of attributes $\pi_{j}$ :

$$
\pi_{j}=1-E_{j}, \quad j=1,2,3, \ldots, n
$$

(3) Calculate objective weight:

$$
\omega_{o b j}^{j}=\frac{\pi_{j}}{\sum_{k=1}^{n} \pi_{k}}, \quad j=1,2,3, \ldots, n
$$

The results of $E, \pi, \omega_{o b j}$ are shown in Table 4 .

(i) Objective Processing. According to research in [29, 30], use the S-type function in the nonlinear model, combining different characteristics of the network attributes, distinguishing 
TABLE 2: The original decision matrix $X$.

\begin{tabular}{|c|c|c|c|c|c|}
\hline Network & Delay (ms) & Jitter (ms) & Throughput (MHz) & Packet loss rate (\%) & Cost \\
\hline UMTS & 15 & 6 & 0.8 & 0.02 & 4 \\
\hline $802.11 \mathrm{a}$ & 29 & 12 & 2.5 & 0.04 & 1 \\
\hline $802.11 \mathrm{~b}$ & 29 & 12 & 2 & 0.04 & 1 \\
\hline WiMAX & 21 & 9 & 2 & 0.03 & 2.5 \\
\hline
\end{tabular}

TABLE 3: The normalized decision matrix $R$.

\begin{tabular}{lccccc}
\hline Network & Delay & Jitter & Throughput & Packet loss rate & Cost \\
\hline UMTS & 0.3096 & 0.2981 & 0.2073 & 0.2981 & 0.8123 \\
\hline $802.11 \mathrm{a}$ & 0.5985 & 0.5963 & 0.6479 & 0.5963 & 0.2031 \\
\hline $802.11 \mathrm{~b}$ & 0.5985 & 0.5963 & 0.5183 & 0.5963 & 0.2031 \\
\hline WiMAX & 0.4334 & 0.4472 & 0.5183 & 0.4472 & 0.5077 \\
\hline
\end{tabular}

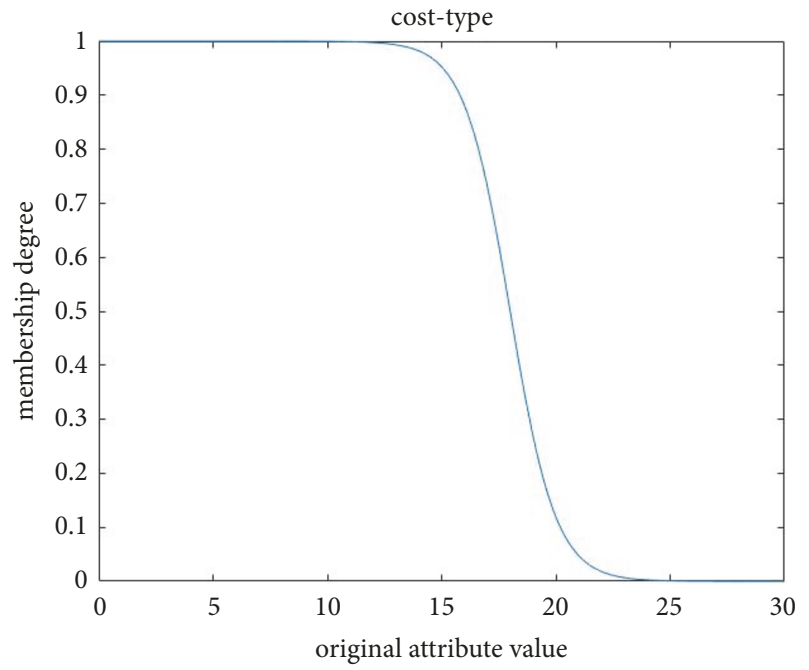

FIGURE 2: Cost-type mapping function.

the cost-type and benefit-type attributes, to determine the two mapping models.

$S_{m j}$ is the standard value of attribute $j$ in business $m$, in which $m=1,2,3,4 ; j=1,2,3,4,5$. Two mapping models are as follows:

Cost-type: $\mu_{i j}=1 /\left(1+\exp \left(x_{i j}-\beta \bullet S_{m j}\right)\right)$, where $\beta=$ 1.2. The graph of function is shown in Figure 2.

Benefit-type: $\mu_{i j}=1-1 /\left(1+\exp \left(x_{i j}-\alpha \cdot S_{m j}\right)\right)$, where $\alpha=0.8$. The graph of function is shown in Figure 3 .

The standard values of different business in Table 5 are also referred to in [27].

After acquiring membership degree from mapping function, according to $\pi_{\beta}=\sqrt{1-\left(\mu_{\beta}\right)^{2}-\left(\nu_{\beta}\right)^{2}}$, the nonmembership degree can be easily resolved. What needs attention is that uncertainty value may be adjusted to $\pi_{p}(x)=$ $\sqrt{1-\mu_{p}^{2}(x)}$, when $\mu_{p}^{2}(x)+\pi_{p}^{2}(x) \geq 1$. Meanwhile, the nonmembership degree $v_{p}(x)$ is zero.

Table 6 shows the final decision matrix results from objective processing. Each member of the matrix is a PFN.

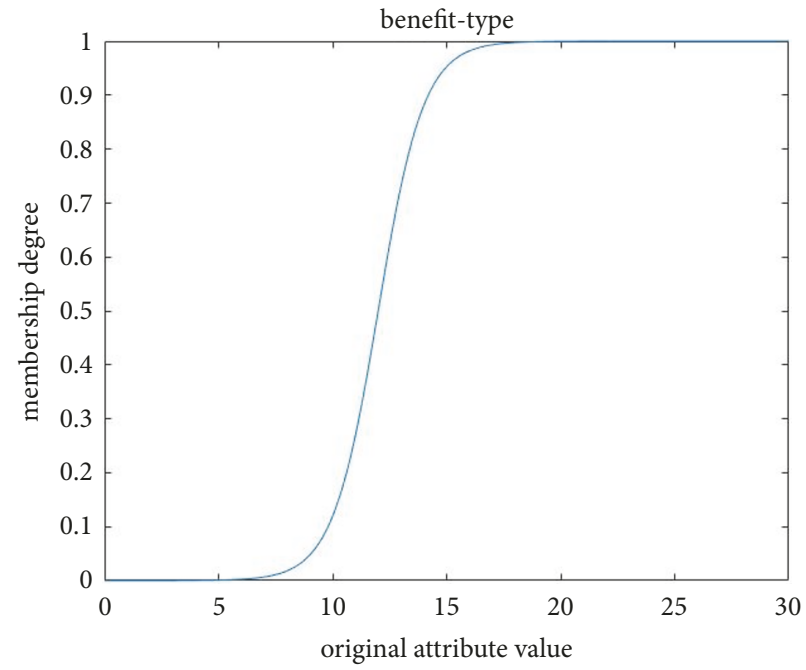

FIgURE 3: Benefit-type mapping function.

(ii) Subjective Processing. According subjective evaluation of experts, we have the final decision matrix 2 in Table 7. Users feedback can also be taken into consideration in practical applications.

As can be seen from Figure 4, two pieces of data generally keep a similar trend while the objective is quite fierce when judging a network, for mapping function chosen is strict due to its high slope which is near the critical value. The subjective method shows more tolerance to networks, for experts have put practical situation into consideration. The similar trend proves the validity of developed objective method. Therefore, both methods are valid and can be applied in this experiment.

It is noteworthy that an objective system established in a specified circumstance needs to be confirmed by traditional methods such as expert scoring, which ensures that it reflects subjective will of people.

(3) Experiment Results. Then, considering different business concerns about network performance distinctively, there exists the possible interaction between these properties. Session business is more sensitive to delay and jitter, while it 
TABLE 4: Calculation results.

\begin{tabular}{lccccc}
\hline Network & Delay & Jitter & Throughput & Packet loss rate & Cost \\
\hline$E$ & 0.9762 & 0.9750 & 0.9513 & 0.9750 & 0.8787 \\
\hline$\pi$ & 0.0238 & 0.0250 & 0.0487 & 0.0250 & 0.1213 \\
\hline$\omega_{\text {obj }}$ & 0.0976 & 0.1024 & 0.2000 & 0.1024 & 0.4975 \\
\hline
\end{tabular}

TABLE 5: Standard value matrix $S_{m j}$.

\begin{tabular}{lccccc}
\hline Business & Delay $(\mathrm{ms})$ & Jitter $(\mathrm{ms})$ & Throughput $(\mathrm{MHZ})$ & Packet loss rate $(\%)$ & 0.02 \\
\hline Session & 15 & 6 & 0.02 & 0.04 & 1 \\
\hline Stream & 30 & 15 & 1 & 0.1 & 0.04 \\
\hline Interactive & 40 & 20 & 0.1 & 0.03 & 1 \\
\hline Background & 100 & 30 & 1 \\
\hline
\end{tabular}

TABLE 6: Final decision matrix $1\left\{\beta_{i j}^{o b j}\right\}$.

\begin{tabular}{lccccr}
\hline Network & Delay & Jitter & Throughput & Packet loss rate & Cost \\
\hline UMTS & $(0.9526,0.3034)$ & $(0.7685,0.6393)$ & $(0.6865,0.7255)$ & $(0.5010,0.8651)$ & $(0.0573,0.9910)$ \\
\hline 802.11a & $(0,0.9997)$ & $(0.0082,0.9997)$ & $(0.9230,0.3817)$ & $(0.4960,0.8680)$ & $(0.5498,0.8264)$ \\
\hline $802.11 \mathrm{~b}$ & $(0,0.9997)$ & $(0.0082,0.9997)$ & $(0.8791,0.4741)$ & $(0.4960,0.8680)$ & $(0.5498,0.8264)$ \\
\hline WiMAX & $(0.0474,0.9986)$ & $(0.1419,0.9896)$ & $(0.8791,0.4741)$ & $(0.4985,0.8665)$ & $(0.2142,0.9692)$ \\
\hline
\end{tabular}

TABLE 7: Final decision matrix $2\left\{\beta_{i j}^{s u b}\right\}$.

\begin{tabular}{lccccc}
\hline Network & Delay & Jitter & Throughput & Packet lose rate & Cost \\
\hline UMTS & $(0.71,0.33)$ & $(0.73,0.31)$ & $(0.90,0.27)$ & $(0.74,0.32)$ & $(0.18,0.84)$ \\
\hline 802.11a & $(0.28,0.77)$ & $(0.24,0.82)$ & $(0.79,0.32)$ & $(0.34,0.62)$ & $(0.73,0.32)$ \\
\hline $802.11 \mathrm{~b}$ & $(0.28,0.77)$ & $(0.24,0.82)$ & $(0.72,0.38)$ & $(0.34,0.62)$ & $(0.73,0.32)$ \\
\hline WiMAX & $(0.43,0.65)$ & $(0.56,0.62)$ & $(0.73,0.36)$ & $(0.45,0.68)$ & $(0.39,0.78)$ \\
\hline
\end{tabular}

is tolerant to packet loss. According to the definition in [24], when $A$ is a subset of $S$, we have

$$
\begin{aligned}
F_{m}(S) & =F_{m}\left(\bigcup_{i=1}^{n} s_{i}\right) \\
& = \begin{cases}\frac{1}{\rho}\left(\prod_{i=1}^{n}\left(1+\rho F_{m}\left(s_{i}\right)\right)-1\right), & \rho \neq 0 \\
\sum_{i=1}^{n} F_{m}\left(s_{i}\right), & \rho=0 .\end{cases} \\
F_{m}(A) & = \begin{cases}\frac{1}{\rho}\left(\prod_{s_{i} \in A}\left(1+\rho F_{m}\left(s_{i}\right)\right)-1\right), & \rho \neq 0 \\
\sum_{s_{i} \in A} F_{m}\left(s_{i}\right), & \rho=0 .\end{cases}
\end{aligned}
$$

For $F_{m}(S)=1$, we can get $\rho+1=\prod_{i=1}^{n}\left(1+\rho F_{m}\left(s_{i}\right)\right)$.

And when we apply the fuzzy measure value which is determined by attribute relationship in Table 8 , it is easily to calculate $\rho=-0.6253$.

According to the above data conditions (Tables 6 and 7), we separately use PFCOWA, PFCWA, PFCWOG, PFCWG, and operators to integrate the attributes information. Both subjective fuzzy value and objective fuzzy value are applied to experiment. Table 9 shows the aggregated results by developed operators, using two kinds of evaluation methods. In Figures 5 and 6 , the results can be compared to each other more intuitively. Table 10 demonstrates the corresponding decision results of subjective and objective evaluation values in different integration modes, comparing with the result of the improved TOPSIS method used in [27].

At first, regardless of which algorithm is used in the context of this experiment, the selection of session services is always UMITS. And the priority of 802.11a network is always higher than that of $802.11 b$. The consistency of these two points confirms the effectiveness of the operators and evaluation system, because the main task in the MADM problems of network selection is to find out the network which has foremost priority.

The main difference in ranking results lies in the evaluation of the WIMAX network. Most of the integration results of the subjective evaluation are the same as those of the TOPSIS method [27]. It is considered that the WIMAX has the priority merely after UMITS. However, in the objective evaluation method, its priority is mostly placed at the end. The reason for this is the characteristics of the mapping function in the objective assessment, which has high slope near critical value. Once it exceeds the critical value, it becomes flat 

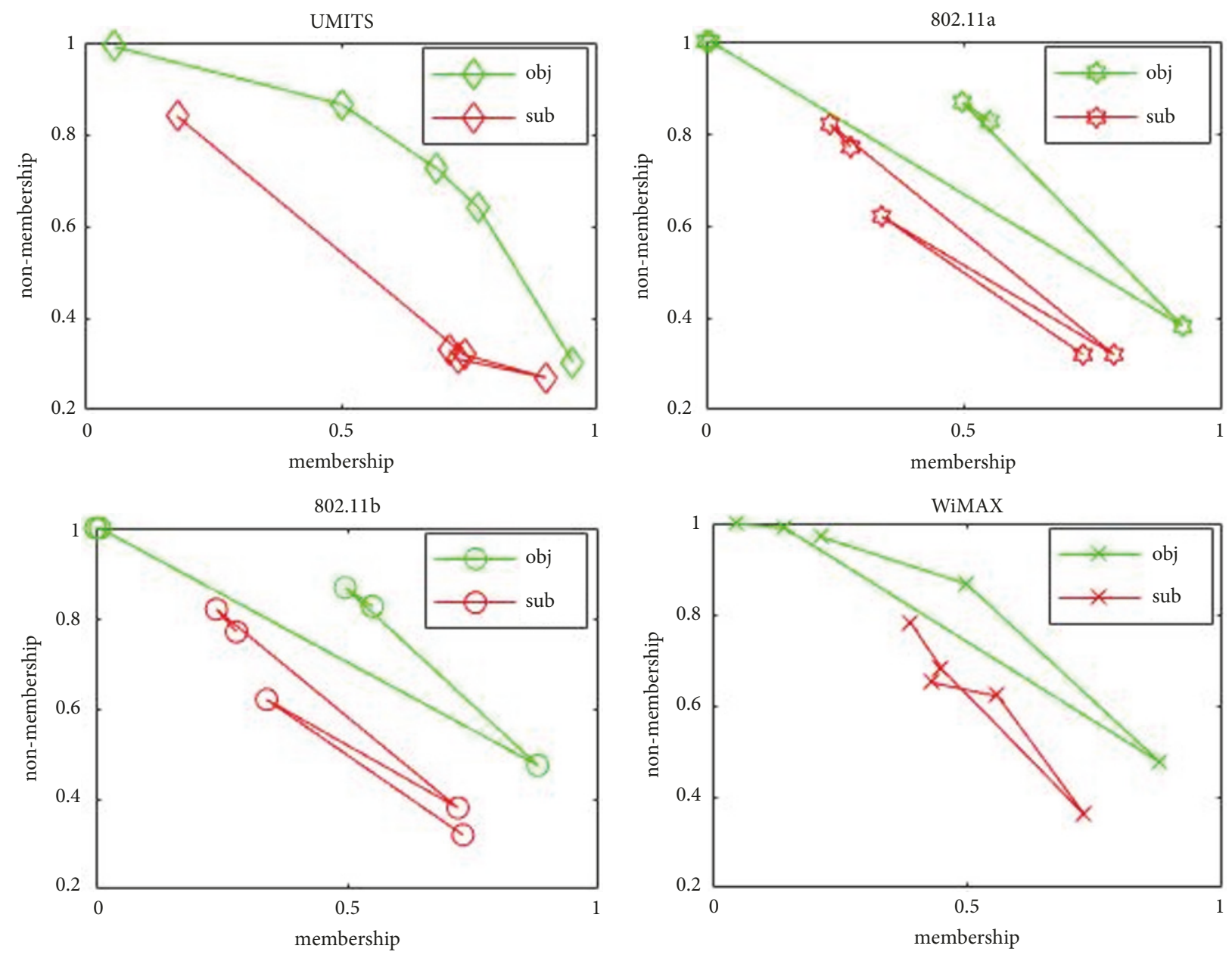

FIgURE 4: Comparison of sub\&obj decision value.

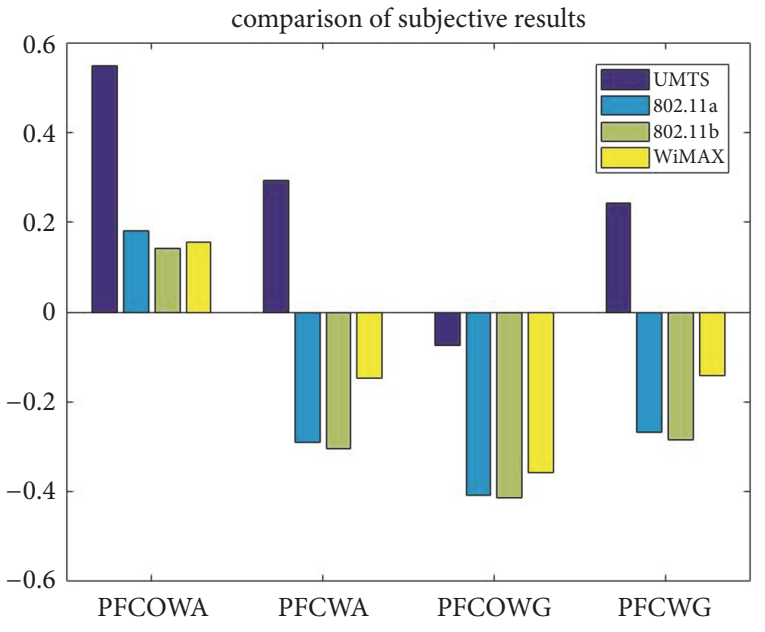

Figure 5: Comparison of subjective results.

rapidly, which no longer has a large discrimination. It brings out a situation when WIMAX performs better than 802.11a and $802.11 \mathrm{~b}$ in many attributes; however, they all exceed the standard value of session business, so these advantages are

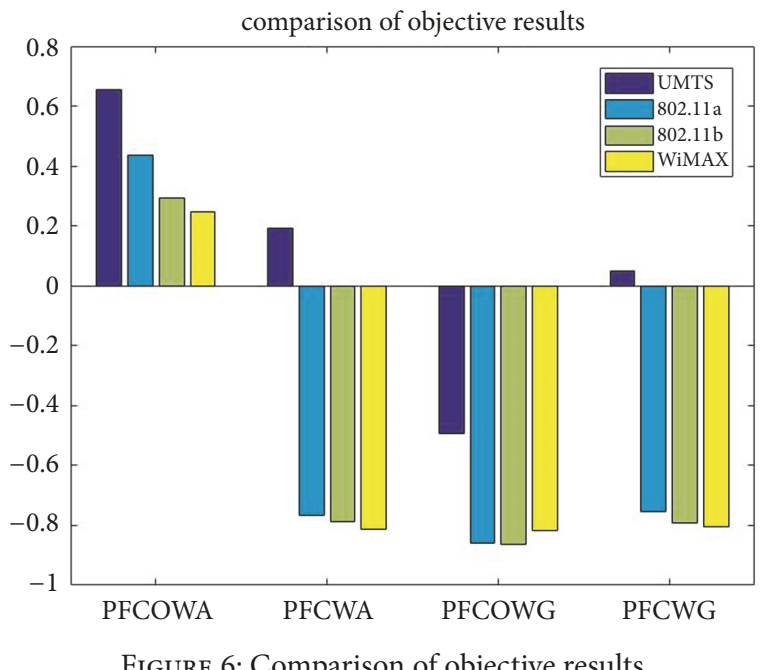

FIGURE 6: Comparison of objective results.

no longer considered, whereas, due to the drawback in the cost attribute, the evaluation of WIMAX in the objective evaluation results is the lowest. 
TAble 8: Fuzzy measure.

\begin{tabular}{llllll}
\hline & $Q_{1}$ & $Q_{2}$ & $Q_{3}$ & $Q_{4}$ & \\
\hline$F_{m}$ & 0.4 & 0.4 & 0.2 & 0.1 & $Q_{5}$ \\
\hline
\end{tabular}

TABLE 9: Aggregated results.

\begin{tabular}{|c|c|c|c|c|}
\hline subjective & PFCOWA & PFCWA & PFCOWG & PFCWG \\
\hline UMTS & $(0.750510 .12425)$ & $(0.665510 .38733)$ & $(0.38890 .47469)$ & $(0.609360 .3598)$ \\
\hline $802.11 \mathrm{a}$ & $(0.530770 .31589)$ & $(0.390580 .66474)$ & $(0.15280 .65733)$ & $(0.346320 .62287)$ \\
\hline $802.11 \mathrm{~b}$ & $(0.497810 .32553)$ & $(0.382710 .67149)$ & $(0.147690 .65913)$ & $(0.342720 .63503)$ \\
\hline WiMAX & $(0.530670 .35575)$ & $(0.498180 .62785)$ & $(0.23820 .64267)$ & $(0.488470 .61634)$ \\
\hline objective & PFCOWA & PFCWA & PFCOWG & PFCWG \\
\hline UMTS & $(0.853910 .26942)$ & $(0.721340 .57441)$ & $(0.319570 .77158)$ & $(0.56530 .51905)$ \\
\hline $802.11 \mathrm{a}$ & $(0.771330 .39978)$ & $(0.206750 .89974)$ & $\left(\begin{array}{ll}0 & 0.92664)\end{array}\right.$ & $\left(\begin{array}{ll}0 & 0.86773)\end{array}\right.$ \\
\hline $802.11 \mathrm{~b}$ & $(0.720630 .47549)$ & $(0.201810 .91013)$ & $\left(\begin{array}{lll}0 & 0.92954)\end{array}\right.$ & $\left(\begin{array}{lll}0 & 0.88914)\end{array}\right.$ \\
\hline WiMAX & $(0.704240 .50083)$ & $(0.214560 .92634)$ & $(0.149080 .917)$ & $\left(\begin{array}{l}0.12652 \\
0.90573)\end{array}\right.$ \\
\hline
\end{tabular}

TABLE 10: Decision strategies by aggregation results by different operators.

\begin{tabular}{lcccc}
\hline Ranking & 1 & 2 & 3 & 4 \\
\hline original & UMTS & WiMAX & $802.11 \mathrm{a}$ & $802.11 \mathrm{~b}$ \\
PFCOWA(sub) & UMTS & $802.11 \mathrm{a}$ & WiMAX & $802.11 \mathrm{~b}$ \\
PFCWA(sub) & UMTS & WiMAX & $802.11 \mathrm{a}$ & $802.11 \mathrm{~b}$ \\
PFCOWG(sub) & WiMAX & $802.11 \mathrm{a}$ & $802.11 \mathrm{~b}$ \\
PFCWG(sub) & UMTS & WiMAX & $802.11 \mathrm{a}$ & $802.11 \mathrm{~b}$ \\
PFCOWA(obj) & UMTS & $802.11 \mathrm{a}$ & $802.11 \mathrm{~b}$ & WiMAX \\
PFCWA(obj) & UMTS & $802.11 \mathrm{a}$ & $802.11 \mathrm{~b}$ & WiMAX \\
PFCOWG(obj) & WiMAX & $802.11 \mathrm{a}$ & $802.11 \mathrm{~b}$ \\
PFCWG(obj) & UMTS & $802.11 \mathrm{a}$ & $802.11 \mathrm{~b}$ & WiMAX \\
\hline
\end{tabular}

This kind of evaluation system is similar to the thought that pass rate prioritizes average score. It is undoubtedly a reasonable idea for the users' quality of experience on network. After comprehensive consideration, the objective evaluation results are scientific and reasonable, which meet the needs of users and can make the same decision as subjective evaluation method does. Moreover, the objective evaluation method has the characteristics of convenience, real-time performance, and high portability. Therefore, in a heterogeneous network environment similar to the experimental environment in this paper, using the proposed QOE objective evaluation system and the proposed operator for MADM problems of network selection is reasonable and effective.

\section{Concluding Remarks}

In this text, some new kinds of aggregation operators are developed. The common character of them is having the ability to put attributes relationship into consideration of decision, which is important for some special scenarios such as network selection in heterogeneous wireless network. Meanwhile, transfer relationship and properties of the operators are given after definitions. Furthermore, an QOE evaluation system is proposed. Participation of Pythagorean fuzzy number makes exacter description possible for QOE value. The novel concept of objective evaluation process provides effectiveness and portability at the same time. A typical example of network selection has proved the validity of both operators and the system. It is convincing to say the study has a bright potential on practical application. What needs to be improved in the future is how to combine the merits of subjective opinion with objective method, representing a more comprehensive result. Moreover, even more complex application scenarios are worth deeper consideration.

\section{Data Availability}

The original data of network performance and business standard are available in [27], and the fuzzy measure data are available in [22]. Beyond that, all the data can be calculated by methods proposed.

\section{Conflicts of Interest}

The authors declare that they have no conflicts of interest.

\section{Acknowledgments}

This work has been partly supported by the National Natural Science Foundation of China [Grants nos. 61702543 and 
71501186] and the 333 High-Level Talent Training project of Jiangsu Province of China [Grant no. BRA 2016542].

\section{References}

[1] K. T. Atanassov, "Intuitionistic fuzzy sets," Fuzzy Sets and Systems, vol. 20, no. 1, pp. 87-96, 1986.

[2] Z. Xu, "Intuitionistic fuzzy aggregation operators," IEEE Transactions on Fuzzy Systems, vol. 15, no. 6, pp. 1179-1187, 2007.

[3] Z. S. Xu and R. R. Yager, "Some geometric aggregation operators based on intuitionistic fuzzy sets," International Journal of General Systems, vol. 35, no. 4, pp. 417-433, 2006.

[4] R. R. Yager and A. M. Abbasov, "Pythagorean membership grades, complex numbers, and decision making," International Journal of Intelligent Systems, vol. 28, no. 5, pp. 436-452, 2013.

[5] R. R. Yager, "Pythagorean fuzzy subsets," in Proceedings of the 9th Joint World Congress on Fuzzy Systems and NAFIPS Annual Meeting, IFSA/NAFIPS 2013, pp. 57-61, Edmonton, Canada, June 2013.

[6] R. R. Yager, "Pythagorean membership grades in multicriteria decision making," IEEE Transactions on Fuzzy Systems, vol. 22, no. 4, pp. 958-965, 2014.

[7] X. L. Zhang and Z. S. Xu, "Extension of TOPSIS to multiple criteria decision making with pythagorean fuzzy sets," International Journal of Intelligent Systems, vol. 29, no. 12, pp. 1061-1078, 2014.

[8] W.-F. Liu, J. Chang, and X. He, "Generalized Pythagorean fuzzy aggregation operators and applications in decision making," Control and Decision, vol. 31, no. 12, pp. 2280-2286, 2016.

[9] X. Zhang, "Multicriteria Pythagorean fuzzy decision analysis: a hierarchical QUALIFLEX approach with the closeness indexbased ranking methods," Information Sciences, 2015.

[10] X. Peng and Y. Yang, "Some results for Pythagorean fuzzy sets," International Journal of Intelligent Systems, vol. 30, no. 11, pp. 1133-1160, 2015.

[11] R. R. Yager, "Properties and applications of Pythagorean fuzzy sets," in Imprecision and Uncertainty in Information Representation and Processing, P. Angelov and S. Sotirov, Eds., vol. 332 of Studies in Fuzziness and Soft Computing, pp. 119-136, Springer, 2016.

[12] S. Dick, R. R. Yager, and O. Yazdanbakhsh, "On pythagorean and complex fuzzy set operations," IEEE Transactions on Fuzzy Systems, vol. 24, no. 5, pp. 1009-1021, 2016.

[13] P. Ji, J. Wang, and H. Zhang, "Frank prioritized Bonferroni mean operator with single-valued neutrosophic sets and its application in selecting third-party logistics providers," Neural Computing and Applications, vol. 30, no. 3, pp. 799-823, 2018.

[14] P. Juan-juan, W. Jian-qiang, and H. Jun-hua, "Multi-criteria decision-making approach based on single-valued neutrosophic hesitant fuzzy geometric weighted choquet integral heronian mean operator," Journal of Intelligent \& Fuzzy Systems: Applications in Engineering and Technology, p. 14, 2018.

[15] R. R. Yager, "Induced aggregation operators," Fuzzy Sets and Systems, vol. 137, no. 1, pp. 59-69, 2003.

[16] R. R. Yager, "Choquet aggregation using order inducing variables," International Journal of Uncertainty, Fuzziness and Knowledge-Based Systems, vol. 12, no. 1, pp. 69-88, 2004.

[17] Z. S. Xu, "Choquet integrals of weighted intuitionistic fuzzy information," Information Sciences, vol. 180, no. 5, pp. 726-736, 2010.
[18] Z. Xu and M. Xia, "Induced generalized intuitionistic fuzzy operators," Knowledge-Based Systems, vol. 24, no. 2, pp. 197-209, 2011.

[19] C. Q. Tan and X. H. Chen, "Induced Choquet ordered averaging operator and its application to group decision making," International Journal of Intelligent Systems, vol. 25, no. 1, pp. 59-82, 2010.

[20] C. Tan and X. Chen, "Induced intuitionistic fuzzy Choquet integral operator for multicriteria decision making," International Journal of Intelligent Systems, vol. 26, no. 7, pp. 659-686, 2011.

[21] G. Choquet, "Theory of capacities," Annales de l'Institut Fourier, vol. 5, pp. 131-295, 1954.

[22] K. X. Cheng, L. Zhu, L. Wang, and X. F. Liang, "Relationship prioritized Pythagorean fuzzy aggregation operators and their application on networks," Acta Technica, vol. 62, pp. 139-148, 2017.

[23] S. Wu, X.-D. Feng, and Z.-G. Shan, "Missing data imputation approach based on incomplete data clustering," Chinese Journal of Computers, vol. 35, no. 8, pp. 1726-1738, 2012.

[24] Z. Y. Wang and G. J. Klir, Fuzzy Measure Theory, Plenum Press, New York, NY, USA, 1992.

[25] Readings in Fuzzy Sets for Intelligent Systems, Elsevier, 1993.

[26] T. Murofushi and M. Sugeno, "Fuzzy measures and fuzzy integrals," in Fuzzy measures and integrals, vol. 40 of Stud. Fuzziness Soft Comput., pp. 3-41, Physica, Heidelberg, 2000.

[27] S. Chen, Research on Network Selection Scheme in Heterogeneous Networks, Beijing University of Posts and Telecommunications, 2010.

[28] D. Zhang, Y. Zhang, N. Lv, and Y. He, "An access selection algorithm based on GRA integrated with FAHP and entropy weight in hybrid wireless environment," in Proceedings of the 2013 7th International Conference on Application of Information and Communication Technologies (AICT), pp. 1-5, Baku, Azerbaijan, October 2013.

[29] H. J. Kim, D. H. Lee, J. M. Lee, K. H. Lee, W. Lyu, and S. G. Choi, "The QoE Evaluation Method through the QoS-QoE Correlation Model," in Proceedings of the 2008 Fourth International Conference on Networked Computing and Advanced Information Management (NCM), pp. 719-725, Gyeongju, South Korea, September 2008.

[30] Y. Zhang, Research on Admission Control Algorithm in Heterogeneous Network Based on QoE, Chongqing University of Posts and Telecommunications, 2016. 


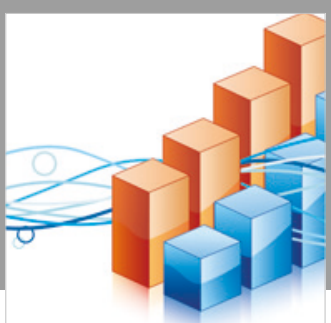

Advances in

Operations Research

\section{-n-m}
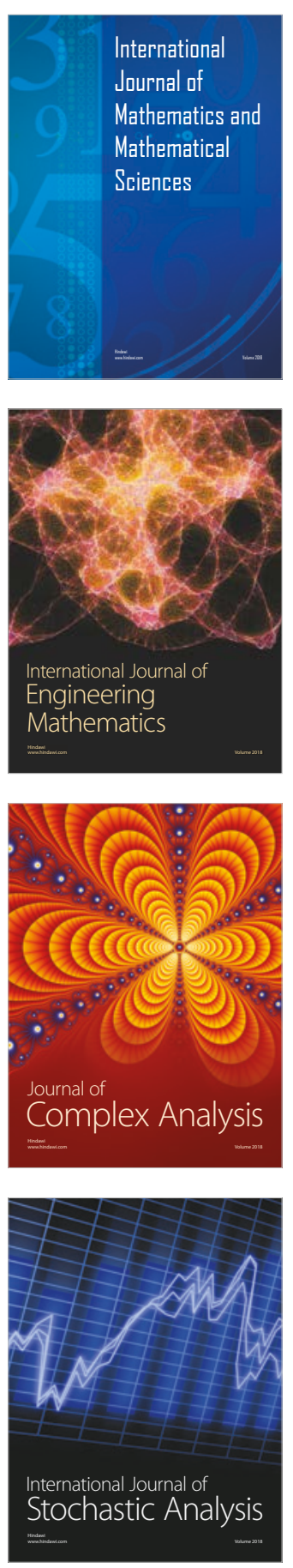
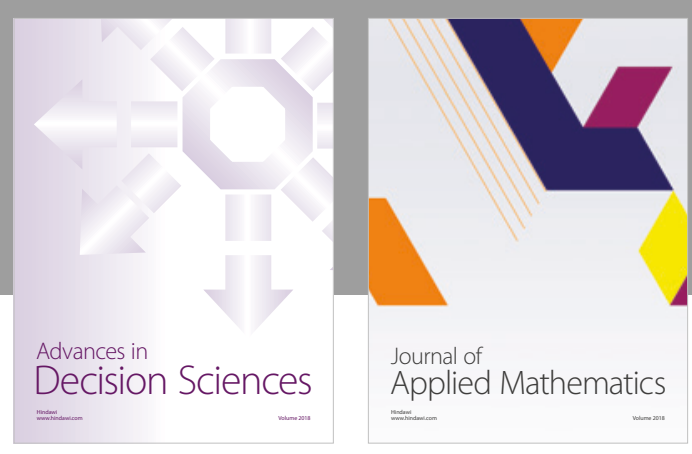

Journal of

Applied Mathematics
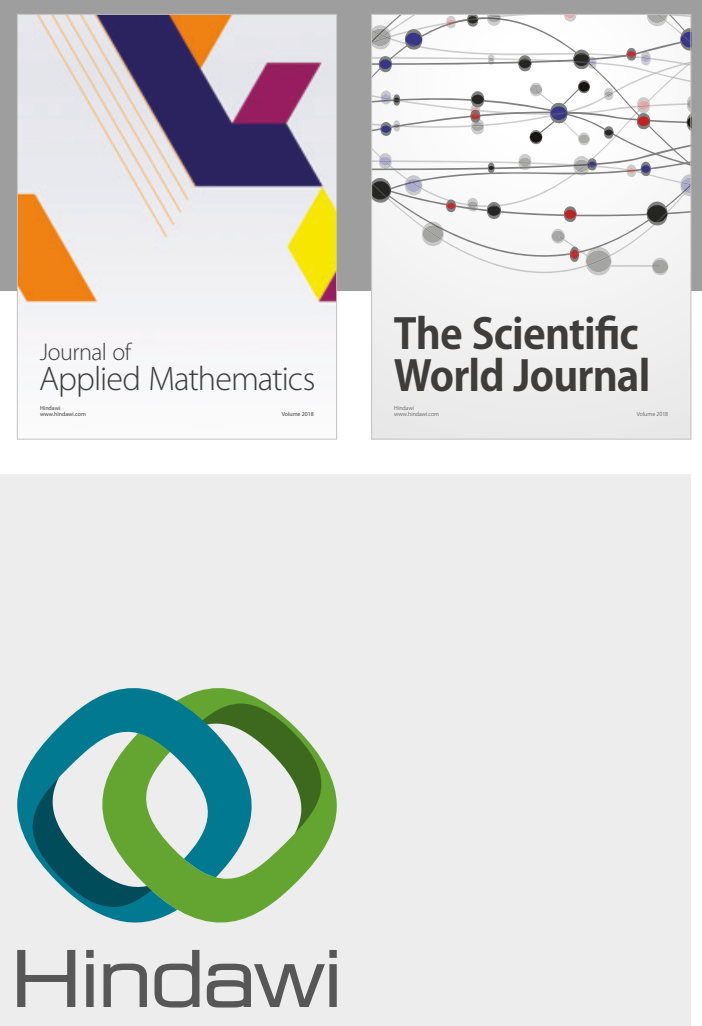

Submit your manuscripts at

www.hindawi.com

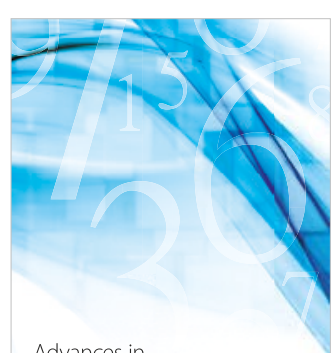

Advances in
Numerical Analysis
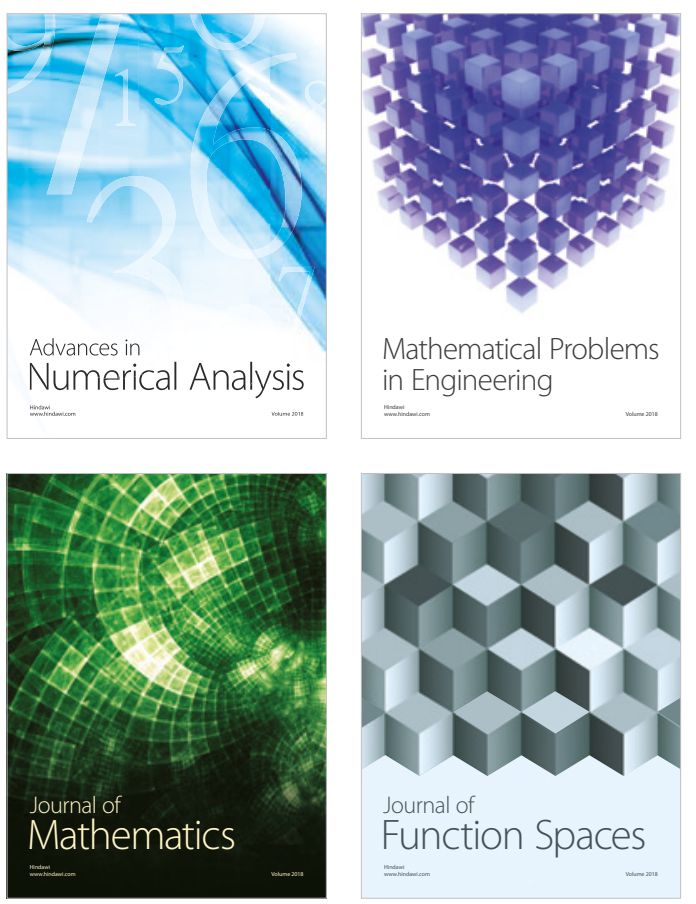

Mathematical Problems in Engineering

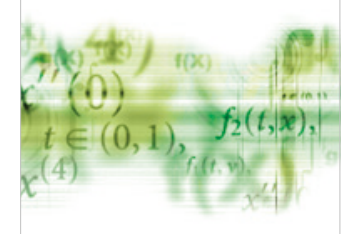

International Journal of

Differential Equations

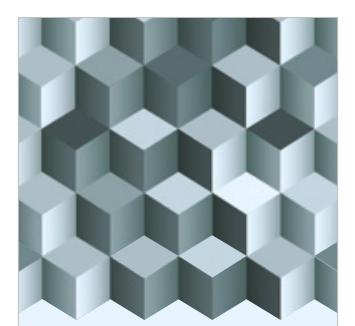

Journal of

Function Spaces

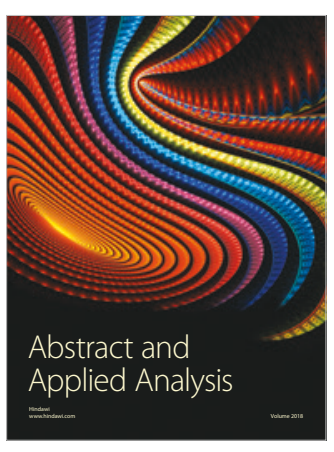

The Scientific

World Journal

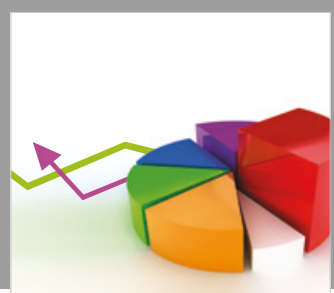

Journal of

Probability and Statistics
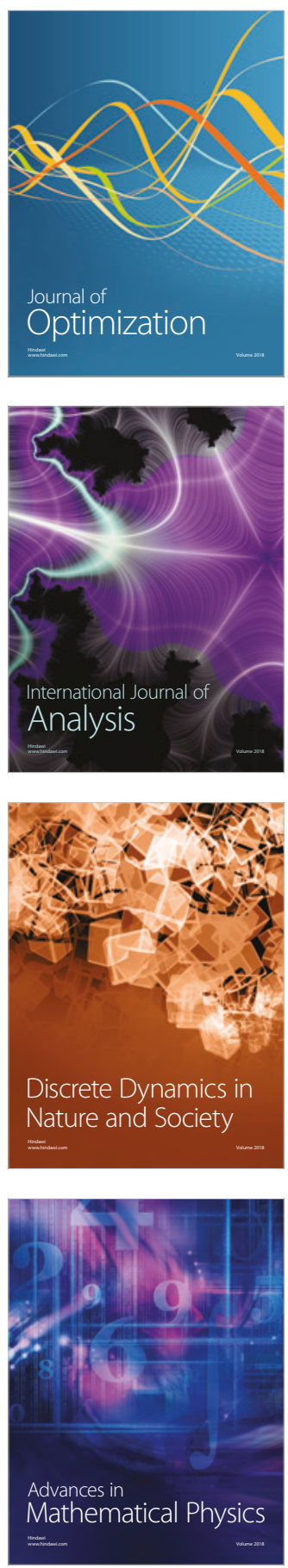